\title{
Increasing the specularity of surface scattering of conduction electrons caused by adsorption of a hydrogen monolayer on the W(110) surface
}

\author{
S.V. Sologub, I.V. Bordenyuk, O.V. Kanash, R.H. Amirov \\ Institute of Physics, National Academy of Sciences of Ukraine \\ 46, prospect Nauky, 03650 Kyiv, Ukraine, e-mail: sologub@iop.kiev.ua
}

\begin{abstract}
Reported in this paper are the results of investigations aimed at scattering of conduction electrons at the tungsten (110) surface covered with hydrogen submonolayers, in which the effect of increasing the specularity of surface scattering of conduction electrons after formation the adsorption phase $\mathrm{W}(110)-(1 \times 1) \mathrm{H}$ has been confirmed. The monolayer coverage with hydrogen was formed as a result of the lowtemperature adsorption involving extrinsic precursor adsorption molecular states. The experiments were carried out using two techniques based on the classical galvanomagnetic size phenomena - the static skin effect and transverse magnetoresistance. It has allowed to confirm the surface nature of the observed effect.
\end{abstract}

Keywords: surface scattering of conduction electrons, surface electron states, adsorption, static skin effect, transverse magnetoresistance.

Manuscript received 02.11.15; revised version received 22.01.16; accepted for publication 16.03.16; published online 08.04.16.

\section{Introduction}

Electron transport in conductors with reduced dimension is the base of functioning for many devices of nanoelectronics and spintronics. In turn, the properties of two surface phenomena - the surface scattering of current carriers [1] and surface electron states [2], interacting between each other, create conditions for the controlled manipulation of properties of low-dimensional systems. In particular, as it was shown in our previous investigations [3, 4], monolayer adsorption of hydrogen on the tungsten and molybdenum (110) surfaces increases the specularity of surface scattering of conduction electrons, which is related to transformation of surface electron structure $[5,6]$. In addition, the method of static skin effect [7] applied in these experiments facilitated increasing the influence of specularity of surface scattering of conduction electrons on the magnetoresistance (MS) of metal plates.
An opinion about impossibility of distinguishing the contribution from surface and bulk electron states to the conductivity of metal objects has been established in the scientific literature [9]. In this paper, we demonstrate that transformation of surface electron structure induced by low-temperature adsorption blocks transitions between surface and bulk electron states and thus allows revealing the role of these surface states in the conductance of low-dimensional metallic systems.

\section{Experimental technique}

The experiments were carried out in the ultra-high vacuum (UHV) glass devices with a residual gas pressure $p \leq 2 \times 10^{-10}$ Torr. The devices with design described in [7] were equipped with hydrogen sources, pressure gauges and internal facilities for UHV pumping. Cooling the samples with liquid helium was carried out through the molybdenum leads passing 
through the glass of a device. The helium cryostat, in which the device was inserted, contained a superconducting magnet-solenoid and/or the Helmholtz coils providing the orthogonal (relatively to the sample surface directions) magnetic field $H \leq 30 \mathrm{kOe}$.

Experimental samples were thin tungsten single crystal plates with a typical size of $4 \times 10 \mathrm{~mm}^{2}$ and a thickness $d \approx 0.1 \mathrm{~mm}$ oriented in the $\mathrm{W}(110)$ plane so that the long side of the sample corresponded to the direction $\langle 100\rangle$. They were prepared by the known technology described in [7]. This technique along with the UHV technology of decarbonization provide the electron mean free path in the bulk of the plate $l_{4.2 \mathrm{~K}} \sim 1 \mathrm{~mm}$ at $T \approx 4.2 \mathrm{~K}$. Changing the sample temperature, that was controlled using a thermocouple WRe5\%-WRe25\%, was provided by electric current flowing through it. Cleaning the surface before deposition of adsorbates was performed with "flash" heating up to $T=2300 \mathrm{~K}$. Measurements of the magnetoresistance (MS) of the plate was always carried out at $T \approx 4.2 \mathrm{~K}$ using the four-point compensation method.

Two classic galvanomagnetic size phenomena static skin effect (SSE) and transverse magnetoresistance (TMR) - are the base of the applied experimental techniques for studying the surface scattering of conduction electrons [7]. Both effects are possible in ultra-pure perfect crystalline metal plates at cryogenic temperatures $(d<<l)$ in the classically strong magnetic field $\mathbf{H}$ $\left(\omega_{c} \tau<<1\right) ; \omega_{c}$ is the cyclotron frequency for conduction electrons in the field $\mathbf{H}, \tau$ - relaxation time for electrons. SSE is observed in a static magnetic field with the directions $\mathbf{H} \perp \mathbf{n}$ and $\mathbf{H} \perp \mathbf{j}$, where $\mathbf{n}$ - normal to the plate surface, $\mathbf{j}$ - density of current flowing along the plates, i.e. the magnetic field is parallel to the plate surface. Under these conditions, (surface) scattering of current carriers near the surface is more frequent than in the bulk, and the electrical current concentrates near the plate surface.

The magnitude of this current is determined by the specularity of surface scattering, which is characterized by the specularity parameter $p$ - ratio of number of electrons reflected specularly to their total number. For providing the TMR conditions, another direction of the constant magnetic field: $\mathbf{H} \| \mathbf{n}$ and $\mathbf{H} \perp \mathbf{j}$ were used, i.e., the magnetic field was perpendicular to the plate surface. In this case, current carriers move along spiral trajectories between the plate surfaces. In the absence of scattering in the bulk and the specular surface reflection of carriers, the plate resistance tends to infinity. In the case of diffuse surface scattering, displacement of axes of spiral trajectories inherent to current carriers (the trajectory 2 in the inset of Fig. 1b) occurs, which contributes to the electric current. The trajectories of movement of current carriers under the conditions of SSE and TMR are schematically shown in the insets of Figs $1 \mathrm{a}$ and $1 \mathrm{~b}$.
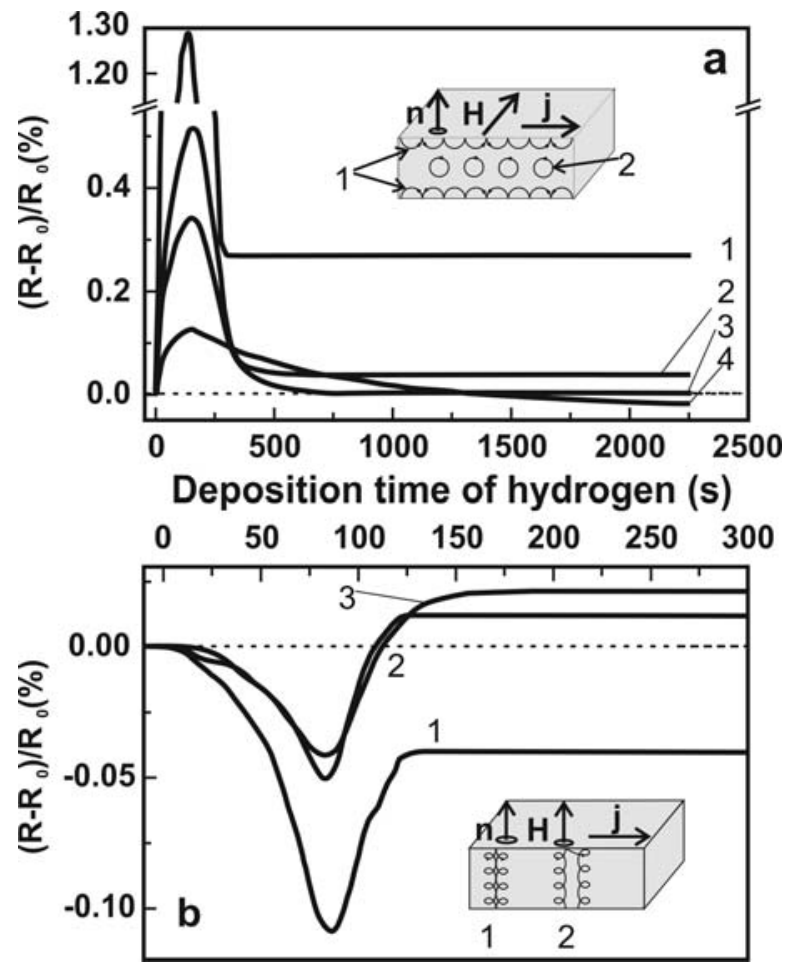

Fig. 1. Changes in the relative MR of the thin $\mathrm{W}(110)$ plate caused by hydrogen adsorption at various temperatures (currents) of the plate. a) SSE $(\mathbf{H} \perp \mathbf{n}): 1-4.6 \mathrm{~K}(1.5 \mathrm{~A})$, 2 - $9.8 \mathrm{~K}(6.0 \mathrm{~A}), 3-10.0 \mathrm{~K}(6.5 \mathrm{~A})$ and $4-14.6 \mathrm{~K}(8.5 \mathrm{~A})$. b) TMR $(\mathbf{H} \| \mathbf{n}): 1-0.2 \mathrm{~A}, 2-1.5 \mathrm{~A}, 3-2.0 \mathrm{~A}$ (in this series of measurements, the temperature is not recorded). In the experiments (a) and (b) we used various samples, the deposition rate of hydrogen was also different. $R_{0}-$ MR value of the plates with atomically clean surface. The insets show configuration of experiments in the modes SSE (a) and TMR (b).

\section{Results and discussion}

Deposition of molecular hydrogen or molecular deuterium on the surface of tungsten (molybdenum) single crystals being under the conditions of SSE at temperatures $T \approx 4.2 \mathrm{~K}$ leads to a bell-shaped change of their magnetoresistance (MR) with reaching the plateau at the end of the curve [7]. This behavior (Fig. 1a) primarily reflects the initial increase in the diffusivity of surface scattering of the conduction electrons of the substrate caused by the increase in the number of adsorbed atoms (adsorption is dissociative) that randomly occupy adsorption sites to achieve the coverage $\theta \approx 0.5$ monolayer (ML). Decreasing MR after the maximum (Fig. 1a) reflects the decrease in diffusivity of surface scattering caused by formation of the partially ordered monolayer of adsorbate $\theta \rightarrow 1$, which restores the natural symmetry of the substrate. In the idealized model, due to equality of the surface concentration of hydrogen adsorption sites and atoms in the topmost layer of the substrate, the structure of hydrogen monolayer lattice completely repeats the 
structure of the topmost layer of the W(110) surface. In accordance with the diffraction concept of the surface scattering of conduction electrons [8], it leads to high specularity of scattering of current carriers on the surface coated with the ideal adsorption phase W(110)- $(1 \times 1) \mathrm{H}$. Since the height of the plateau at the end of the MC curves (Fig. 1a) characterizes the specularity of scattering of current carriers by this structure, this MR value characterizes the degree of its ordering.

Fig. 1a also shows the change in MR of W(110) plate due to adsorption of hydrogen measured at its various temperatures close to the temperature of liquid helium. Increasing the adsorption temperature during adsorption leads to the following changes in the MR curves:

a) The maximum in the MR curves decreases [10] It is associated with increasing scattering of conduction electrons by phonons and therefore reduction of their mean free path $l$ in the bulk of W. In turn, under the SSE condition it means increasing the conductivity of the plate bulk and "shunting" the conductivity of the surface region. Reducing $l$ also leads to decrease in the nearsurface conductivity itself, the value of which represents the degree of specularity of the surface scattering of conduction electrons.

b) The width of the MR curves, more precisely, the length of their falling part increases. It is related with the mechanism of formation of the hydrogen monolayer with participation of extrinsic precursor adsorption states [7]. Before reaching coverage with hydrogen $\theta \approx 0.5 \mathrm{ML}$, atoms of hydrogen randomly fill the adsorption sites on the W(110) surface. With increasing the coverage, the probability of formation of twodimensional clusters of adatoms increases. On the surface of these clusters, the hydrogen molecules can be captured into the so-called extrinsic precursor adsorption states (Fig. 2a). Diffusion of physadsorbed molecules to the boundaries of clusters leads to dissociation of the molecules on the bare tungsten surface and adsorption of atoms in the nearest adsorption sites. Temperature increasing leads to increasing the rate of molecular diffusion, and hence, the increasing rate of formation of monolayer atomic coverage with hydrogen. Nevertheless, further increasing the temperature reduces the probability of capturing the molecules into precursor states and results in slowing down W(110)- $(1 \times 1) \mathrm{H}$ phase formation. The latter process is manifested via increasing the time of atomic monolayer formation with increasing the temperature (Fig. 1a) ("tightening" the descending part of the curves) [7].

c) The height of the MR plateau corresponding to formation of hydrogen monolayer coverage decreases with increasing the substrate temperature and, in the end, the plateau falls down even lower than for the plate with atomically clean surfaces. This result confirms the effect that we observed earlier and which was induced by formation of an ordered monolayer of hydrogen via high-temperature $(T=200-300 \mathrm{~K})$ annealing of adsorbate films deposited up to saturation at $T \approx 4.2 \mathrm{~K}$ on the surfaces W(110) [3] and Mo(110) [4].

The surface nature of this effect is confirmed by the results of another series of experiments (Fig. 1b), in which the influence of submonolayer adsorption of hydrogen on MR of the W(110) plate being in the TMR conditions $(\mathbf{H} \| \mathbf{n})$ was studied. While maintaining the basic qualitative peculiarities inherent to the curves in Fig. 1a, the curves of Fig. 1b have the opposite sign of effect and less absolute value. For clarity, we note that in this series of experiments (Fig. 1b) the plate temperature was not directly measured, and the hydrogen pressure during deposition, apparently, was growing, as evidenced by the "tightened" initial part of the curves and their shape more "compressed" as compared to Fig. 1a.

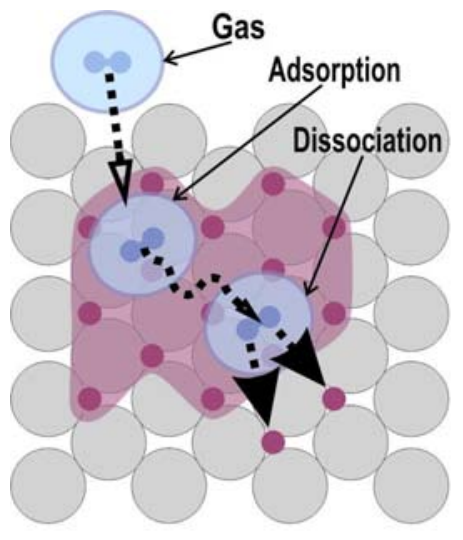

a

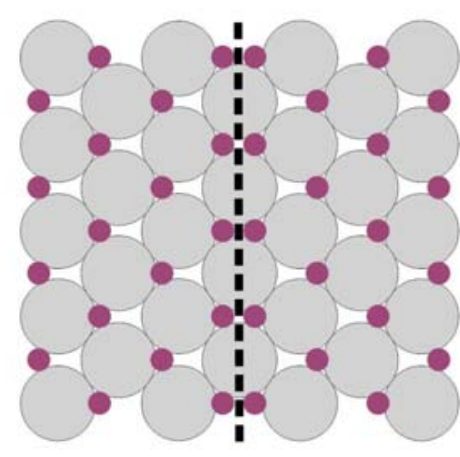

b

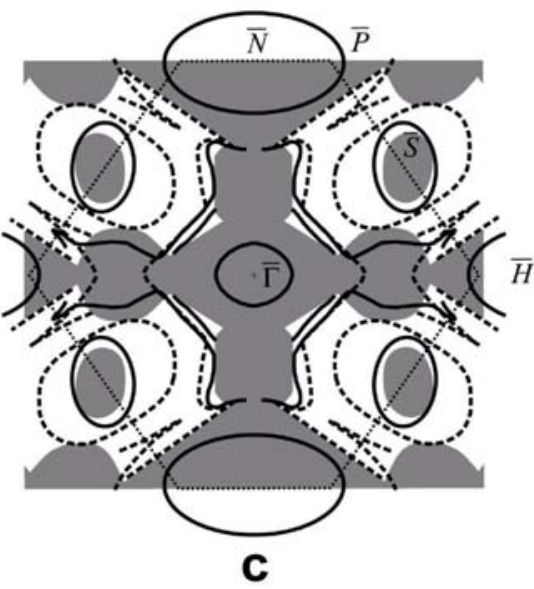

Fig. 2. a) model of dissociative adsorption of hydrogen with participation of extrinsic preadsorption states: hydrogen molecule from the gas phase is captured into prestates on the surface of the island of adsorbed atoms, diffuses to the borders of the island and dissociates with subsequent adsorption of atoms; b) two types of domains in a monolayer of hydrogen adsorbed on the W(110) surface: large circles - tungsten, small ones - hydrogen; c) shadow projection of Fermi surface of tungsten bulk (filled area) and the structure of the surface electronic states on W(110) surface [5]: solid lines - atomically clean surface, dashed lines - the surface covered with ordered monolayer of hydrogen shown in (b). 
Under TMR conditions (Fig. 1b), the increase of surface scattering due to increasing the concentration of adsorbed hydrogen $(0 \leq \theta \leq 0.5 \mathrm{ML})$ leads to displacement of axes of spiral orbits of electrons passing between two surfaces of the plate (the inset in Fig. 1b), and hence, to reduction of its resistance. Similarly, the increase in specularity of surface scattering during formation of the adsorbate monolayer $(0.5 \leq \theta \leq 1 \mathrm{ML})$ leads to a decrease in the displacement of axes of spiral electron orbits and increase of the resistance. However, the most important (for this investigation) feature of the curves in Fig. $1 \mathrm{~b}$ is an increase of the MR at plateau, corresponding to formation of hydrogen monolayer, to the value higher than for the atomic clean surface, which is manifested at elevated temperature (higher current) of the substrate. This is the result of the same effect that was observed under SSE conditions (Fig. 1a).

In two series of the experiments carried out under the TMR and SSE conditions, the direction of the magnetic field $\mathbf{H}$ differs by $90^{\circ}$, but the $\mathbf{H}$ direction is always perpendicular to the direction of the current $\mathbf{j}$, flowing along the plate, i.e., $\langle 100\rangle$ direction that is the axis of 4-th order of the $\mathrm{W}$ single crystal. Therefore, these two directions of the magnetic field are physically equivalent for the properties of the $\mathrm{W}$ crystal bulk. That's why, a different sign of the effects that were observed under the TMR and SSE conditions reflects the change in surface properties, namely, change in the character of the surface scattering of current carriers. The dependences of Fig. 1b are "mirror image" of the curves in Fig. 1a.

The physical picture of increasing the specularity of conduction electrons scattering, which was caused by monolayer hydrogen adsorption on the W(110), was considered in our previous papers [3, 4]. To explain the effect transformation of the surface electron structure of atomically clean surface of W(110) plate induced by adsorption of an ordered hydrogen monolayer, which was measured by angle-resolved photoemission spectroscopy method (Fig. 2c), should be considered [5, $6]$. This figure shows a shadow projection of the Fermi surface of bulk electron states of tungsten on the plane (110) as well as projections of Fermi tubes (Fermi contours) of surface electron states of atomically clean and covered with hydrogen monolayer of the W(110) surface. It is obvious that the hallmark of transformation of the electron structure of the surface induced by adsorption is the disappearance of one group of Fermi contours that were superimposed on the projection of the "bulk" part of the Fermi surface and "squeezing" the another group of these contours beyond its boundaries of the "bulk" part. Within the frame of the diffractional concept [8], the scattering of the conduction electrons by the metallic crystal surface occurs in accordance with the conservation laws of energy $\varepsilon$ and the tangential component of the quasi-momentum $\mathbf{k}_{t}$ :

$\mathbf{k}_{t^{\prime}}=\mathbf{k}_{t}+n \mathbf{g}$,

$\varepsilon^{\prime}(\mathbf{k})=\varepsilon(\mathbf{k})=\varepsilon_{\mathrm{F}}$, where $\varepsilon_{\mathrm{F}}-$ Fermi energy, $n$ - an arbitrary integer, $\mathbf{g}$ - an arbitrary vector of the reciprocal surface lattice, and hatched and unhatched values correspond to the state of electron before and after scattering. In accordance with Eqs (1) and (2), overlapping projections of tungsten Fermi surface (FS) parts, corresponding to bulk and surface electron states, which is attributed to the atomically clean W(110) surface, opens the channels for transitions between these groups of electron states, and these transitions can be realized during the surface scattering of current carriers. In this case, the conservation law of the tangential component of the quasi-momentum (1) is valid for $n=0$, the so-called "vertical" transitions of current carriers occur, and the conservation law for energy (2) is valid automatically, since electrons of bulk and surface states are in thermodynamical equilibrium and possess the Fermi energy $\varepsilon_{\mathrm{F}} \pm k_{\mathrm{B}} T$, where $k_{\mathrm{B}}$ is the Boltzmann constant.

The transitions between the surface and bulk electron states strongly change the direction of the group velocity of the conduction electrons. Transformation of the surface electron structure caused by formation of the $\mathrm{W}(110)-(1 \times 1) \mathrm{H}$ adsorption phase leads to a strong reduction of the channels for transitions between the surface and bulk electron states and, consequently, increases the specularity of the surface scattering of current carriers.

In conclusion, we note that the found effect is observed on the background of the factors that increase the surface scattering of electrons, particularly, different scattering cross sections of conduction electrons by tungsten atoms and hydrogen adatoms, shift the hydrogen monolayer lattice relative to the surface lattice of tungsten, as well as the presence of two types of domains of adsorbed hydrogen in the W(110)- $(1 \times 1) \mathrm{H}$ surface phase, which provoke the scattering of conduction electrons by the domain walls (Fig. 1b). It means that the value of "pure" effect, in fact, is even greater. In addition, we note that the lack of surface reconstruction under the hydrogen adsorption on the tungsten (110) surface also contributes to the manifestation of this effect [11].

\section{Conclusions}

In the present study of scattering of conduction electrons on atomically clean and covered with hydrogen submonolayer W(110) surface, the effect of increasing the specularity of surface scattering of conduction electrons under formation of the W(110)- $(1 \times 1) \mathrm{H}$ adsorption phase has been confirmed. The reason of the effect is in significant reduction of the transition channels between the surface and bulk electron states as a result of transformation of surface electron structure of $\mathrm{W}(110)$, which was induced by monolayer adsorption of hydrogen. Unlike our previous experiments [3, 4], in which the monolayer coverage of hydrogen adatoms on the W(110) surface was formed via high-temperature 
( $T=200-300 \mathrm{~K})$ annealing of saturated hydrogen coverage deposited at helium temperature, in these experiments the adsorbate monolayer was formed via low-temperature adsorption with participation of extrinsic precursor adsorption molecular states. Application in the experiments of two methods based on the classic galvanomagnetic size phenomena - static skin effect and transverse magnetoresistance - made it possible to confirm the surface nature of the observed effect.

\section{References}

1. V.F. Gantmakher, Y.B. Levinson, Carrier Scattering in Metals and Semiconductors. Modern Problems in Condensed Matter Sciences, Vol. 19. North-Holland, Oxford, New York, Tokio, 1987.

2. A. Zangwill, Physics at Surfaces. Cambrige University Press, New York, 1988, Pt. 1, Chapt. 4.

3. O.A. Panchenko, and S.V. Sologub, Effect of surface states on the surface scattering of current carriers // Phys. Rev. B, 71, 193401 (2005).

4. O.A. Panchenko, S.V. Sologub, I.V. Bordenjuk, Scattering of current carriers on a Mo(110) surface covered with hydrogen and deuterium submonolayers // Surf. Sci. 605, p. 1287 (2011).
5. E. Rotenberg and S.D. Kevan, Evolution of the electronic and structural properties of W(011) with H coverage // Phys. Rev. Lett. 80, p. 2905 (1998).

6. K. Jeong, R.H. Gaylord, S.D. Kevan, Experimental Fermi surface of Mo(011) // Phys. Rev. B, 39, p. 2973 (1989).

7. O.A. Panchenko, P.P. Lutsishin, S.V. Sologub, Galvanomagnetic size studies of metallic surface processes // Progr. Surf. Sci. 69, p. 193 (2002).

8. A.F. Andreev, Interaction of conducting electrons with the metal surface // Uspekhi fizicheskikh nauk, 105, p. 113-1243 (1971), in Russian.

9. Ph. Hofmann, J.W. Wells, Surface-sensitive conductance measurements // J. Phys.: Condens. Matter, 21, 013003 (21 p.) (2009).

10. A.A. Mitryaev, O.A. Panchenko, G.M. Pugach, Temperature dependence of the resistivity of thin tungsten plates in a strong magnetic field // Sov. Phys. JETP, 48(4), p. 683-686 (1978).

11. M. Arnold, S. Sologub, G. Hupfauer, P. Bayer, W. Frie, L. Hammer, K. Heinz, LEED structure analyses of clean and fully hydrogen covered W(110) and Mo(110) surfaces // Surf. Rev. \& Lett. 4, p. 1291 (1997). 